\title{
A comparison between parallelization approaches in molecular dynamics simulations on GPUs
}

\author{
Lorenzo Rovigatti, ${ }^{1,2, a)}$ Petr Šulc, ${ }^{3}$ István Z. Reguly, ${ }^{4}$ and Flavio Romano ${ }^{5}$ \\ ${ }^{1)}$ Dipartimento di Fisica, Sapienza-Università di Roma, Piazzale A. Moro 5, 00185 Roma, \\ Italy \\ ${ }^{2)}$ Faculty of Physics, University of Vienna, Boltzmanngasse 5, A-1090 Vienna, \\ Austria \\ ${ }^{3)}$ Rudolf Peierls Centre for Theoretical Physics, University of Oxford, 1 Keble Road, Oxford, OX1 3NP, \\ United Kingdom \\ 4) Oxford e-Research Centre, University of Oxford, Oxford, 7 Keble Road, Oxford, OX1 3QG, \\ United Kingdom \\ ${ }^{5)}$ Physical $\& 3$ Theoretical Chemistry Laboratory, Department of Chemistry, University of Oxford, South Parks Road, \\ Oxford, OX1 3QZ, United Kingdom
}

(Dated: 6 November 2018)

\begin{abstract}
We test the relative performances of two different approaches to the computation of forces for molecular dynamics simulations on Graphics Processing Units. A "vertex-based" approach, where a computing thread is started per particle, is compared to an "edge-based" approach, where a thread is started per each potentially non-zero interaction. We find that the former is more efficient for systems with many simple interactions per particle, while the latter is more efficient if the system has more complicated interactions or fewer of them. By comparing computation times on more and less recent GPU technology, we predict that, if the current trend of increasing the number of processing cores - as opposed to their computing power - remains, the "edge-based" approach will gradually become the most efficient choice in an increasing number of cases.
\end{abstract}

\section{INTRODUCTION}

For a long time the steady increase in frequency of CPUs guaranteed an almost equally steady increase in the performance of compute-intensive applications with very little effort on the programming side. However, by 2005 , it became clear that, essentially due to power dissipation constraints, this trend would not last for much longer. In order to keep increasing the computational capacity, multi-core CPUs have appeared, the size of on-chip caches has grown and the complexity of control circuitry has increased. At the same time, new architectures have emerged; Graphical Processing Units (GPUs), originally special-purpose hardware for graphics, were equipped to carry out general purpose computations. While most CPU architectures are still aimed at low-latency handling of a few processes, GPUs support a massive amount of parallelism, giving up low latency in exchange for high throughput. By integrating up to thousands of simple computing units, grouping them under just a few dozen control units and connecting them to high-speed memory, they are capable of supporting up to tens or hundreds of thousands of threads executing concurrently. This enables GPUs to deliver an order of magnitude higher performance than CPUs on workloads with a high amount of data parallelism, i.e. where the same operations are carried out over different data. Efficient use of this hardware requires employing parallel programming models that are very explicit about data parallelism and often expose low-level features of the

\footnotetext{
a) Author for correspondence, lorenzo.rovigatti@uniroma1.it
}

GPU. The situation is further complicated by significant architectural changes when a new generation of GPUs is released: improved hardware and new features may affect the relative performance of different algorithms, often permitting some of them to run more efficiently and slowing down others.

Computer simulations are a very valuable tool in many areas of science ${ }^{1-6}$ The relatively cheap computer power offered by GPUs is very attractive to simulators, since it allows extended time scales and large system sizes

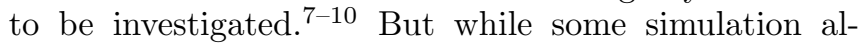
gorithms are relatively easy to parallelize, some others are very difficult to code efficiently on a parallel machine. Molecular dynamics (MD), in its many variants, is a prominent technique in computational physics and chemistry, and it is in principle an algorithm that is suitable for parallelization. ${ }^{4|5| 9 \mid 11}$ Most free and commercial simulation packages have the option to run in parallel, and a growing number of them offers the option to run on GPUs ${ }^{12}$ 16 The different simulation packages exploit GPUs in different ways, each using a distinct approach to overcome the two main obstacles to make full use of a GPU: potentially concurrent writes to the same memory location and having a large number of balanced tasks for the GPU to carry out.

An important difference between CPUs and GPUs is that the latter have a much smaller cache, and therefore the optimisation of memory access patterns has received a lot of attention; since many scientific computations are bound by the amount of data they have to move, it is crucial to achieve as high a bandwidth as possible.

GROMACS 4.6, for example, uses the GPUs for the calculation of non-bonded interactions $\sqrt{17}$ and a parallel reduction algorithm to add the calculated forces be- 
tween pairs of particles, rearranging particles in memory to speed-up memory access. NAMD 15 also implements a GPU-based calculation of non-bonded forces, $\frac{18}{\text { where }}$ forces for each pair of interacting particles are calculated twice. HOOMD-blue ${ }^{12}$ and LAMMPS 13 also compute forces for each interacting pair twice to avoid atomic operations or memory synchronization bottlenecks.

Different parallelization approaches of the MD algorithm have been considered for various platforms. Three main parallel decomposition schemes typically considered are! 11

- Atom-decomposition. The particles in the simulation are split between the computing units, which calculate the interaction forces between them.

- Force-decomposition. The interactions between particles are split in a way that each computing unit is responsible for a particular subset of forces between particles to be evaluated.

- Space-decomposition. The domain of the simulated system is partitioned into smaller subsets, with each computing unit responsible for calculation of forces and updates of positions of particles in its assigned subdomain.

Each of the proposed schemes requires communication between the computing units. The choice of the optimal parallelization scheme hence depends on the system studied as well as on the properties of the hardware architecture. The communication cost and the load balance between computing units then determine the optimal parallelization scheme.

In this work, we implement and study the atom-decomposition approach (which we call the "vertex-based" approach) and a variant of the forcedecomposition approach (which we call the "edge-based" approach) on GPUs, where one computing unit corresponds to a single thread of the graphics card that will be carrying out the computations. In the "vertex-based" approach, a computing thread is started per each particle and the force $\mathbf{F}_{i j}$ between each pair of particles $i$ and $j$ is calculated separately for $i$ and $j$. This might seem like a waste of computational resources, since it is known by Newton's third law that $\mathbf{F}_{i j}=-\mathbf{F}_{j i}$, but it has the advantage of being more parallelizable. In the Supplementary Information we show that often the work saved by using Newton's third law is outbalanced by the fact that the force calculation is less parallel, and in all cases the performances of the two approaches are similar. In the "edge-based" approach, a thread is started per each potentially non-zero interaction, and we use atomic operations and Newton's third law, $\mathbf{F}_{i j}=-\mathbf{F}_{j i}$, to calculate the resulting force acting on each particle.

We study the two parallelization approaches by implementing a GPU-based MD algorithm for three different model molecules (Lennard-Jones particles, patchy particles and coarse-grained DNA, see Section III that differ substantially in the complexity and physical features of the interaction potential and we report the relative performance of the algorithms for different hardware.

To our best knowledge, the edge-based approach has not yet been systematically compared to the vertexbased approach on GPU. Most GPU implementations use the atom-decomposition (i.e., vertex-based in our terminology) scheme.10|12|13|18|19 Zhmurov and collaborators considered both atom and force decomposition schemes for an MD study of a coarse-grained polymer model on $\mathrm{GPU}^{20}$, but a comparison of the performance of the two approaches was not reported.

Interestingly, we find that the edge-based approach holds better performance on newer hardware for shortranged, anisotropically interacting systems. This broad class of coarse-grained models has recently gained significant attention from the soft matter and biophysics community as a tool for the investigation of biological macromolecules 2122 and self-assembling processes $23 \mid 24$ and for the synthesis of new materials 2526 . The performance boost provided by the edge-approach, which can be readily implemented in any modern MD package, will help in exploiting the power of the GPUs in this field with even more efficiency.

\section{METHODS}

For our performance tests, we perform Brownian dynamics simulations in the NVT ensemble, with the thermostat described in Ref. 27. Thermostating the system with this method does not significantly affect performances, and thus we do not expect this thermostat to bias our comparison in any way. We implement a combination of fairly standard optimizations in order to speedup performance. We use Verlet lists ${ }^{4}$ to provide each particle $i$ with a list $L_{i}$ of all the particles inside a sphere centred on $i$ of radius $r_{v}=r_{c}+r_{s}$, where $r_{c}$ is the interaction cut-off and $r_{s}$ is the Verlet skin. Verlet lists are then updated by using a standard cell algorithm. On GPUs, both cell filling and Verlet lists updating are performed on a one-particle-per-thread basis. We perform simulations at different numbers of particles $N$ (nucleotides in the case of the DNA model).

In the case of the Lennard-Jones and patchy models (see Section III for details), we maximise cache hits by periodically sorting particle data, stored in the global memory, according to a 8 -vertex Hilbert curve.12 The resulting speed-up depends on $N$, ranging from $20 \%$ to $100 \%$ for $N>10^{4}$ as discussed in Ref. 28. We do not apply this procedure to DNA simulations, since doing so does not result in any significant measurable gain.

One of the potential drawbacks of using GPUs is in the accuracy of floating point operations. Even though double precision support is quickly improving, the GPUs' peak double precision performance is only a half or a third of its peak single precision performance, and it is thus crucial to use single-precision performance as much as possible. Unfortunately, it has been shown 
that lengthy single precision simulations lack reliability even for simple potentials. ${ }^{29}$ In order to maximise performances and minimise numerical instabilities, we use double precision calculations to carry out the integration of positions and momenta and single precision calculations to compute forces, as commonly done in many MD packages. This mixed precision algorithm results in a performance decrease ranging between $10 \%$ and $40 \%$ compared to single precision (depending on the model and simulation parameters), but dramatically improves the numerical stability! 28

We now describe in more detail the two parallelization approaches used in this work. A cartoon providing a visual explanation of the two algorithms is shown in 1 . The first one, which we call "vertex-based", is to start a thread for each of the particles in the system. This thread will go through a list of potentially interacting neighbours, compute the force coming from each of the neighbours, and then add them together to yield the total force acting on the particle. The threads done in this way are completely independent, since they do not need to write concurrently to the same memory.

The second parallelization approach we consider is a more aggressively parallel "edge-based" approach, where a thread is started for each potentially non-zero interaction (or equivalently for each potentially interacting pair of particles). Therefore, the total number of threads is equal to the sum of the number of neighbours of each particle divided by two. Each thread will compute the force due to a specific pair interaction and add it to the total force acting on both particles $i$ and $j$. However, multiple threads trying to concurrently apply a force to the same particle pose a data race which has to be resolved: the most general approach is for the force computation to store the three components of force vectors on a per-edge basis, and once all the threads have finished, carry out a parallel segmented reduction to add up the contributions from each interaction for each particle. The second approach is to use atomic operations to directly accumulate the total force acting on a particle. However, compared to regular memory transactions, atomic operations are very expensive. Therefore the update should not be performed naively, but only if the force is actually non-zero. This is a common occurrence when treating anisotropic interactions, since it is possible to have particles separated by a distance $r<r_{c}$ but mutually oriented in such a way that the force acting between them is zero, but is also relatively common when treating isotropic interactions since the Verlet lists always contain a significant fraction of non-interacting particles. Threads computing an interaction between two particles that turns out to be zero thus do not carry out any atomic operations. We stress that in this approach it is natural to exploit Newton's third law, since many different threads compute the forces acting on a single particle and thus threads cannot be independent by design.

The vertex-based approach has the advantage of being "embarrassingly parallel"; threads do not have to communicate or synchronize in any way. On the other hand, the amount of threads that get started at the same time is equal to the number of particles in the system, which poses a lower bound on the computer time required per step: if there are fewer particles than the number of threads required to saturate the GPU (we point out that the current trend is to increase the number of concurrent threads), there will be no computational benefit in studying smaller systems using the vertex-based approach. We also point out that the edge-based approach is only effective if treating systems with short-range forces (i.e., forces that vanish faster than $r^{-3}$ in $3 D$ ), since otherwise $\mathcal{O}\left(N^{2}\right)$ threads would need to be started. While this approach is in principle feasible for small values of $N$, the many concurrent updates to the force vector acting on each particle would make it not competitive.

It is important to identify the bottlenecks in each of the approaches, since the convenience in using one parallelization approach or the other will depend on how each particular case is affected by these bottlenecks. The vertex-based approach is limited by insufficient parallelism at low $N$, and by the computational time required by the slowest thread in each of the warps, and thus by the computation of the forces of the particle that has the most and/or most complicated interactions. The edgebased approach, on the other hand, is limited by the most expensive pair interaction, a much lower bound, and by the throughput of atomic operations.

It may be worth addressing in more detail whether it would be worth to exploit Newton's third law $\left(\mathbf{F}_{i j}=\right.$ $\left.-\mathbf{F}_{j i}\right)$ in the vertex-based approach. As discussed earlier, this would half the total number of computations. However, additional operations are then necessary to update the total force acting on the chosen particle. For this reason, many MD packages on GPUs prefer to repeat the calculation to avoid concurrent writes, which are known to be a potential source of slowdown! 10|12|18|20 To provide a quantitative analysis of this point, we also implemented a version of the vertex-based approach that uses Newton's third law. Atomic addition is used to update the total force acting on a particle. In order to avoid most of the slowdown related to the atomic addition of the forces, each particle has $m$ force vectors on which the atomic adds are performed separately, and then the vectors are combined to obtain the total force acting on each particle. The performances turned out to be rather insensitive on the value of $m$, as long as $m>10$. We found that for some of the studied systems the vertex based-approach with Newton's third law was marginally (at most 1.2 times) faster, and for the remaining cases considered its performance was the same or worse than for the vertex-based approach which did not exploit Newton's third law. Importantly, in all cases where the vertex-based approach with Newton's third law is the fastest, it is always slower than the edge-based approach. We hence consider the vertex-based approach without the Newton's third law here and provide benchmarks comparing the vertex-based approaches with and 

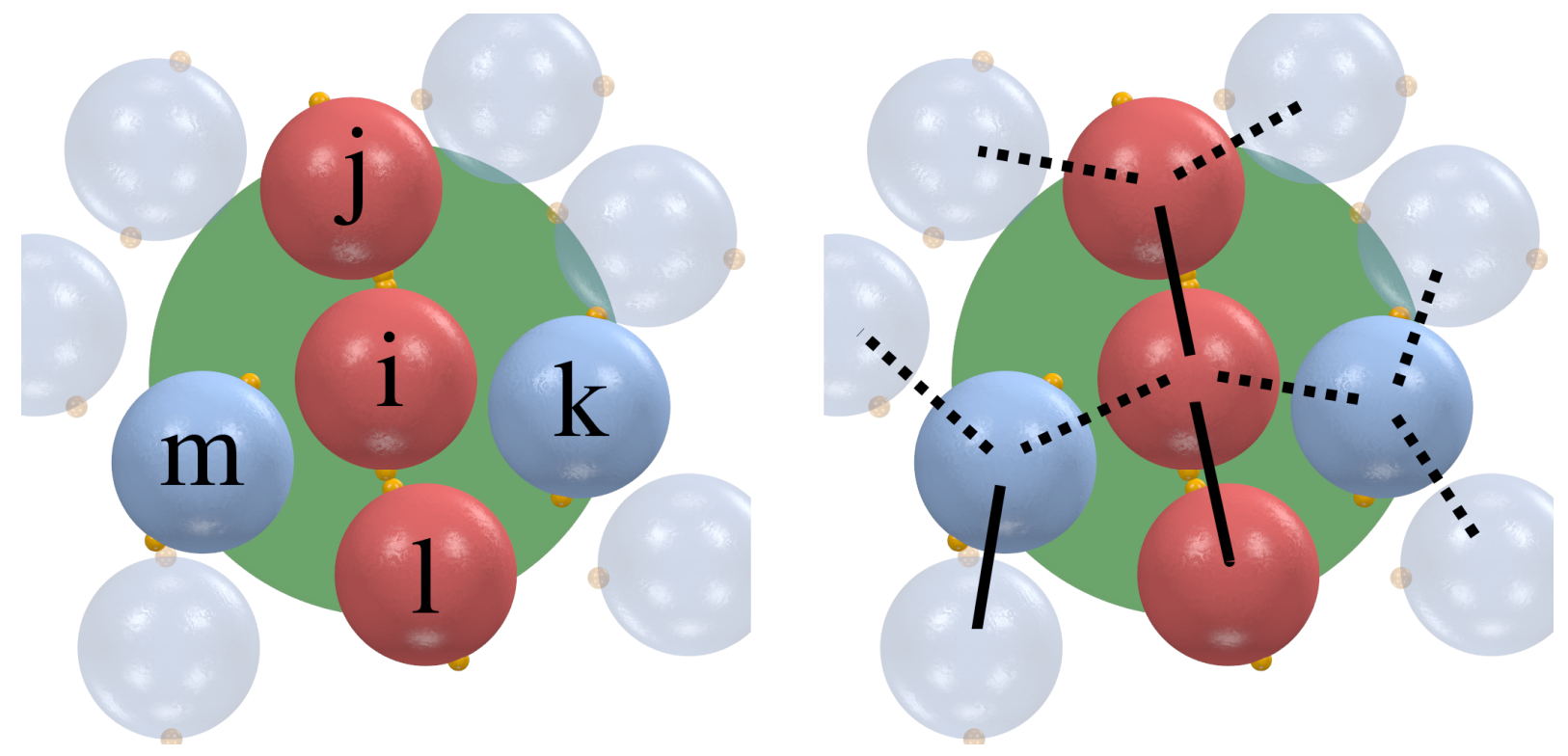

FIG. 1. A cartoon showing how the (left) vertex and (right) edge algorithms work in a patchy system. Patches are depicted as yellow spheres. The Verlet list of the central particle $i$ contains all the particles which are within a sphere of radius $r_{v}$ (in green), $j, k, l$ and $m . i$ and the only two particles exerting a non-zero interaction on it are colour-coded in red, while all the other particles are coloured in light blue. Particles whose centres are outside the Verlet sphere are semitransparent. In the vertex approach (left panel), the $i$-th thread computes the interaction between $i$ and each of the particles in its Verlet list. Thus, if we consider only particles within the green sphere, 5 threads are started. By contrast, in the edge approach (right panel) a thread is started for each potential interaction between two particles, i.e. one for each of the lines connecting particle pairs in the figure. If, once again, we consider only particles within the green sphere, the total number of started threads is 10 , doubling the number of threads of the vertex case and thus enhancing parallelism. The only threads that require to perform expensive atomic operations to update the forces acting on the particles are those for which these forces are different from zero (full lines). In anisotropic models such as the patchy one presented here, a large part of the potential interactions is effectively zero (dashed lines) and hence does not result in any concurrent memory write.

without Newton's third law in the Figure S2 in the Supplementary Information. $\frac{30}{}$

All our simulations have been carried out on CUDAenabled NVIDIA GPUs with oxDNA, a simulation software originally developed to simulate a coarse-grained DNA model ${ }^{31 \sqrt[32]{32}}$ now extended to support additional interaction potentials. The code is open source and can be freely downloaded from the oxDNA website $\underline{33}$

It is important to ensure that our algorithm comparisons are carried out with a simulation code of good overall efficiency, otherwise the results would be of little use. We thus compared the oxDNA implementation of vertexbased parallelization for Lennard-Jones (LJ) systems, a very common test potential, consisting of up to 262144 particles with two popular simulation packages that also allow for MD simulations on GPUs: LAMMPS13 (1Feb14 version) and HOOMD-blue (0.11-3 version) $!^{[12}$ Figure 2 shows some benchmarks, obtained on Tesla C2070 and Kepler K10 GPU cards. The speed of our code is within $10 \%$ of the performance of the HOOMD-blue package for systems composed of 10000 particles or more, being slightly faster for larger systems. In comparison with LAMMPS, our code is at least $20 \%$ faster for all the systems considered. More details can be found in the Sup-

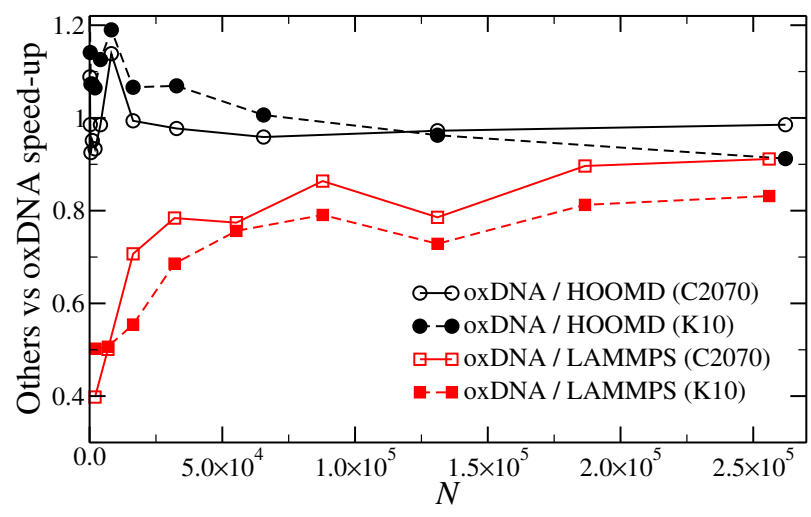

FIG. 2. Relative performance of oxDNA vs LAMMPS (in red) and HOOMD-blue (in black) for different system sizes on Tesla C2070 (full lines, empty symbols) and Kepler K10 (dashed lines, full symbols) GPUs. A y-value larger (smaller) than one means that oxDNA is slower (faster) than the other package.

plementary Information (Fig. S1) a performance comparison between oxDNA and GROMACS 5.0.1 on a LJ system showing that oxDNA is 
much faster than GROMACS in this case. We thus confirm that our implementation of the MD algorithm has performances comparable with two widely used parallel MD simulation packages oriented towards the soft matter community.

We note that GPU vs CPU relative performance depends dramatically on the hardware and even more on the simulation parameters and system under study. Common speed-ups for LJ and patchy systems of a single GPU vs a single CPU-core for large systems varies between factors of 20 and 100, depending mainly on the density. ${ }^{12,29,41}$ As for the DNA model, the performance gain is usually between 20 and 50.28

\section{MODELS}

In order to compare the performance of the two parallelization approaches on diverse situations, we have implemented three different models with substantially different features and different scopes. The first model is the widely employed Lennard-Jones interaction potential, used to model atomic and molecular systems such as noble gases and glass-forming materials! 434 This iconic model potential, often used to test algorithms, is a pairwise, spherically symmetric potential that can be written in terms of the relative distance $r$ between two particles as

$$
V(r)=4 \epsilon\left[\left(\frac{\sigma}{r}\right)^{12}-\left(\frac{\sigma}{r}\right)^{6}\right]
$$

where $\epsilon$ controls the depth of the attraction and $\sigma$ is the particle diameter. As commonly done, we cut and shift the potential at a distance $r_{c}=2.5 \sigma$ [4]

The second interaction potential we implement, the patchy model, depends on the relative orientations of each pair of particles as well as their relative distance. Anisotropically interacting systems such as this one are becoming increasingly popular in the soft matter field, due to the richness of phenomena they exhibit ${ }^{35}$ and to the possibility of synthesising particles with tunable shape and surface patterns ${ }^{36}$ Patchy particles are spherical colloids, i.e. nano- or micro-sized particles, with interacting spots decorating their surface. Lately, patchy particles have been the subject of several theoretical, numerical and experimental studies, and have been shown to exhibit novel and unexpected behaviour such as the formation of stress-yielding, density-vanishing equilibrium gels, 27|37 crystallisation into open lattices $25 \mid 38$ or re-entrant gas-liquid phase separations. ${ }^{39 / 40}$ More specifically, we use an interaction potential that comprises a spherical hard-core-like repulsion and a short-ranged interaction that depends on the relative orientations of each pair of particles:

$$
V(1,2)=V_{C M}(1,2)+V_{P}(1,2)
$$

where $V_{C M}$ is the interaction between the centres of mass and $V_{P}$ is the interaction between the patches, modelled as follows:

$$
\begin{aligned}
V_{C M}(12) & =\left(\frac{\sigma}{r_{12}}\right)^{200} \\
V_{P}(12) & =-\sum_{i=1}^{M} \sum_{j=1}^{M} \epsilon \exp \left[\frac{1}{2}\left(\frac{r_{12}^{i j}}{0.12 \sigma}\right)^{n}\right]
\end{aligned}
$$

where $r_{12}$ is the distance between the centres of mass, $r_{12}^{i j}$ is the distance between patch $i$ on particle 1 and patch $j$ on particle 2 and $M$ is the number of patches per particle, which we fix to the value $M=2$. This potential has been used in the past to study the dynamics of patchy particles by means of MD simulations.27/41

The third and last model we use is oxDNA, a coarsegrained model specifically designed to reproduce the mechanical, structural and thermodynamic properties of DNA targeted to simulating processes occurring in DNA nanotechnology $31 / 32 / 42$ Indeed, oxDNA has been used to investigate DNA nanotweezers, $\frac{43}{4}$ DNA walkers, $\frac{44}{,}$, a burnt-bridges DNA motor ${ }^{45}$ and other DNA motifs common in DNA nanotechnology ${ }^{22}$ By exploiting GPUs, oxDNA can be used to investigate systems composed of thousands of nucleotides. 26]46 The basic unit of the oxDNA model is a nucleotide, which is modelled as a rigid body interacting with other nucleotides through a short-ranged, highly anisotropic potential that takes into account contributions due to excluded volume, backbone, stacking, coaxial stacking, cross-stacking and hydrogen bonding interactions. The detailed form of the potential can be found in Refs. 31 and 42 .

From the point of view of the computational complexity, these three models have different properties. In the LJ model, which is spherically symmetric and relatively long-ranged $\left(r_{\mathrm{c}}=2.5 \sigma\right)$, particles can have a large number of interacting neighbours. The patchy model, on the other hand, is very short-ranged $\left(r_{c}=0.18 \sigma\right.$ in our case), and thus each particle has a small number of potentially interacting neighbours, and on top of that its anisotropic nature makes it so that each particle has an interaction which is non-zero with only a fraction of its neighbours. Differently from the other two models, oxDNA features a very complicated and computationally demanding potential that, due to the large amount of branching and imbalance in the calculations required by particle pairs in different local environments, hinders performances on GPUs. Indeed, the GPU vs CPU speed-up for oxDNA is usually smaller than what we find for the LJ or patchy model. Similarly to the patchy model, the high degree of anisotropy of the potential results in a small number of interacting neighbouring particles.

The LJ and patchy models are simulated at three different values of the number density, namely $\rho \sigma^{3}=0.1$, 0.3 and 0.5 The simulation temperature is $k_{B} T / \epsilon=1.8$ for the LJ model and $k_{B} T / \epsilon=0.15$ for the patchy model. 

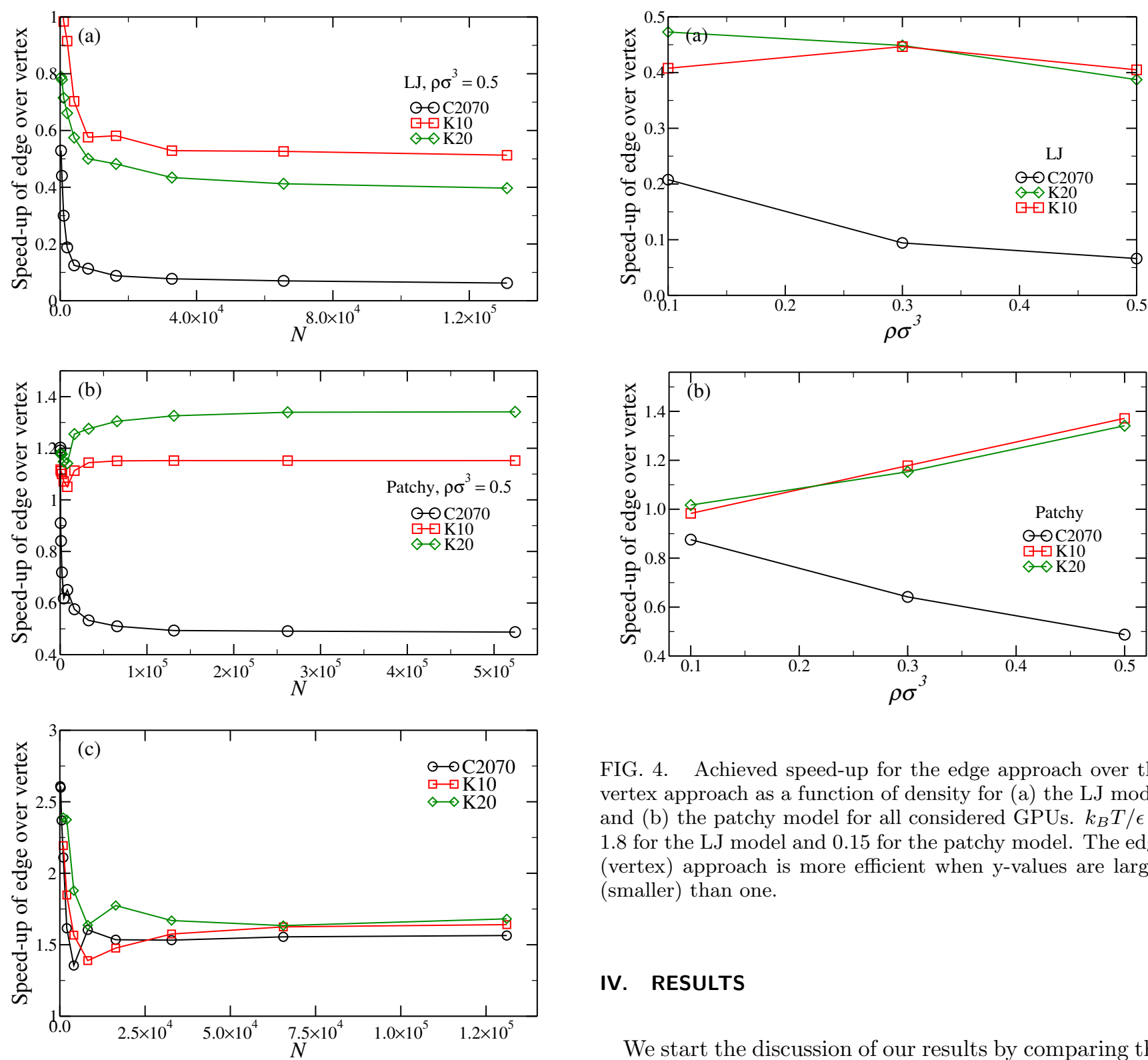

FIG. 4. Achieved speed-up for the edge approach over the vertex approach as a function of density for (a) the LJ model and (b) the patchy model for all considered GPUs. $k_{B} T / \epsilon=$ 1.8 for the LJ model and 0.15 for the patchy model. The edge (vertex) approach is more efficient when $\mathrm{y}$-values are larger (smaller) than one.

\section{RESULTS}

We start the discussion of our results by comparing the performances of the two approaches for the three different models under standard thermodynamic conditions.

FIG. 3. Achieved speed-up for the edge approach over the vertex approach for (a) the LJ model and (b) the patchy model at $\rho \sigma^{3}=0.5$ and (c) for the oxDNA model as a function of the number of particles $N$ and for different GPUs. All bumps and spikes are reproducible. The edge (vertex) approach is more efficient when $\mathrm{y}$-values are larger (smaller) than one.

For the DNA model we perform simulations of doublestranded octamers at a concentration of $2.7 \mathrm{mM}$ and at a temperature of $300 \mathrm{~K}$. chitectures, since these are under active development and tests might give substantially different results. We run our tests on the NVIDIA cards C2070 (Tesla architecture, released in 2010), K10 and K20 (Kepler architecture, released in 2012). In Fig. 3 we report the speed-up due to the edge-based approach versus the vertex-based approach for the patchy and LJ models at the highest density $\rho \sigma^{3}=0.5$ and for oxDNA at the only investigated density. The speed-up is defined as the average running time for the vertex-based algorithm divided by the average running time of the edge-based algorithm. The averages are taken over the same number of cycles with the two algorithms on the same hardware. Each amount of cycles is larger than the decorrelation time of each system, thus giving timings that are reflective of its physical properties. We point out that newer hardware has 
better performance than old hardware in all cases if the same algorithm and model are employed. The timings are repeated 5 times for each data point to accumulate averages. Since floating point operations are not commutative, and the order of operations cannot be controlled in an efficient parallel algorithm, simulations on GPUs (as well as parallel simulations on CPUs) are not reproducible. We thus have to let each simulation undertake a different trajectory and make sure that our timings are taken over long enough intervals to average out the small differences in running time due to the different sequences of calculations.

All panels in Fig. 3 show a plateau in the speed-up for a large enough system, and the height of this plateau is the benchmark that we use to asses the performance of the algorithms. Since GPUs become faster than a single CPU core only at large $(N \gtrsim 500-1000)$ system sizes, we assume that it is most relevant to compare GPU codes for large systems. In the case of the LJ potential, shown in Fig. 3(a), the vertex-based approach is the fastest for all GPUs considered, although newer architectures suffer less from the introduction of the edge-based approach. This is because the edge-based approach is slowed down by carrying out many atomic operations, which are faster in the newest architecture but not yet fast enough to make it competitive. The patchy model displays a different behaviour (Fig. 3(b)): the edge approach becomes favourable on the most recent architecture by almost $40 \%$, compared to a slowdown of $50 \%$ on the oldest hardware. It is the substantially improved speed of atomic operations on the newer hardware architecture that favours the edge-based approach $\stackrel{47}{47}$ The amount of pair interaction computations is essentially controlled by the density, while the number of non-zero interactions has a maximum which is dictated by the interaction potential. This means that the number of interactions that need to be computed and the number of interactions that are actually non-zero both increase with density, but the former increases faster than the latter. Slower atomic operations and a smaller amount of compute units on the older architecture favour the vertex-based approach, that requires fewer threads and no atomic operations. The edge-based approach is faster on the newer hardware because it can better exploit the larger number of threads and is slowed down less by the atomic operations which it requires.

Finally, the compute-intensive pair interactions of the oxDNA model (Fig. 3(c)) always benefit from the edgebased approach, which yields a $70 \%$ performance increase or more with the newest K20 hardware. ${ }^{47}$ There are several reasons for this, the main one being that the vertex approach has a poorer balance between the workload each thread has to carry out as opposed to the edgebased approach, which is itself not very well balanced but hides the load imbalance with a much larger amounts of threads. It is worth pointing out that in general the more recent the hardware the bigger the speed-up of the edgebased approach, because it benefits from more compute units and faster atomic operations. $\underline{47}$
The relative effectiveness of the two parallelization approaches depends on the amount of potentially interacting neighbours. For the LJ and patchy models this number can change significantly as the density of the system is changed, and we thus repeat our tests at three different values of the density. In the DNA model, since the local environment of the molecules stays the same, the density does not change significantly the number of potential interactions and hence performances are very weakly density-dependent, at least in the density range usually considered in DNA applications. The density dependence of the speed-up is shown in Fig. 4 for the three models. In the case of LJ, the relative performances stay more or less constant, except for the oldest hardware where the increased number of atomic operations has a negative impact on the edge-based approach. In the case of the patchy system, on the other hand, increasing the density favours the edge-based approach if using new hardware and favours the vertex-based approach on the older hardware. This can be rationalized as follows: the number of potential interactions increases faster with increasing density than the number of non-zero interactions. The edge-based approach is effective in treating potential interactions that turn out to be zero, because they do not produce atomic operations. But the latter nevertheless increase with density, and when using the oldest hardware the balance is still reversed in favour of the vertex-based approach.

We note that the vertex-based approach reported in this section does not use Newton's third law. We also measured the performance of the vertex-based approach which uses Newton's third law, as outlined in Sec. II. We found its performance to be inferior for LJ systems in comparison with vertex-based approach without Newton's third law on all considered architectures. For the patchy particle systems, we found that on the Kepler architectures K10 and K20 the vertex-based approach with Newton's third law can be faster by up to a factor 1.2 , but it is always slower than the edge-based approach. For the DNA systems, we found the vertex-based approach with Newton's third law to be slightly faster than the approach without Newton's third law on both Tesla and Kepler architectures. However, the achieved speedup was only at most $16 \%$, significantly smaller than the speed-up achieved with the edge-based approach. For completeness, we provide the comparisons of the vertexbased approaches with and without Newton's third law in Supplementary Information (Fig. S2), along with the absolute time per MD step for the results shown in Figure 330

\section{CONCLUSIONS}

Being able to exploit the impressive computer power of GPUs can be important in molecular simulations, since these computing devices have the power to study system sizes and time scales previously untreatable. Unfortu- 
nately, this comes at the cost of rethinking the structure of the simulation codes, since approaches that are known to fail in CPU programming can turn out to be effective or vice-versa. We have compared the performances of two different parallelization approaches, "vertex"- and "edge"-based, by simulating three different models with quite distinct computational complexities. The edgebased parallelization approach, where a thread is started for each potentially non-zero interaction, is competitive and often outperforms the vertex-based approach, where a thread is started for each particle in the system. The reason for this is that sometimes the vertex-based approach is not parallel enough to take full advantage of the GPU.

A vertex-based approach is still the fastest when dealing with very simple potentials with a relatively large number of neighbours, which is the case when the interaction range is large and the interaction is spherical. The edge-based approach is the fastest when the non-zero interactions per particle are few and/or complicated, giving its best performance when the interaction potentials are both highly anisotropic and complicated as it is the case in the oxDNA model. Not surprisingly, the edgebased approach benefits more from an increased number of scalar processors on the graphics card, which appears to be the current trend in improving this kind of hardware. We thus predict that the edge-based parallelization will become more and more competitive in the future, if the current trends in hardware improvements are continued.

\section{ACKNOWLEDGEMENTS}

The authors thank M. Sega for technical help, the Advanced Research Computing, University of Oxford for computer time and NVIDIA for the hardware donations. F.R. acknowledges financial support from the Engineering and Physical Sciences Research Council. P. ̌́. is grateful for the Bobby Berman and Scatcherd European Scholarship awards. L.R. thanks the Physical \& Theoretical Chemistry Laboratory, University of Oxford for its hospitality and acknowledges support from ERC-226207PATCHYCOLLOIDS.

${ }^{1}$ R. Feynman, Int. J. Theor. Phys. 21, 467 (1982)

${ }^{2}$ M. S. Waterman et al., Introduction to computational biology: maps, sequences and genomes. (Chapman \& Hall Ltd, 1995).

${ }^{3} \mathrm{~F}$. Jensen, Introduction to computational chemistry (Wiley, 2007).

${ }^{4}$ B. Smith and D. Frenkel, Understanding molecular simulation (Academic Press, 1996).

${ }^{5}$ M. P. Allen and D. J. Tildesley, Computer simulation of liquids (Oxford University Press, 1989).

${ }^{6}$ D. Landau and K. Binder, A Guide to Monte Carlo Simulations in Statistical Physics (Cambridge University Press, 2005).

${ }^{7}$ J. D. Owens, M. Houston, D. Luebke, S. Green, J. E. Stone, and J. C. Phillips, Proc. IEEE 96, 879 (2008).

${ }^{8}$ J. Nickolls and W. J. Dally, IEEE micro 30, 56 (2010).

${ }^{9}$ J. E. Stone, D. J. Hardy, I. S. Ufimtsev, and K. Schulten, J. Mol. Graphics Modell. 29, 116 (2010)
${ }^{10}$ J. A. van Meel, A. Arnold, D. Frenkel, S. F. Portegies Zwart, and R. G. Belleman, Mol. Simulat. 34, 259 (2008).

${ }^{11}$ S. Plimpton, J. Comput. Phys. 117, 1 (1995).

12 J. A. Anderson, C. D. Lorenz, and A. Travesset, J. Comput. Phys. 227, 5342 (2008).

${ }^{13}$ W. M. Brown, P. Wang, S. J. Plimpton, and A. N. Tharrington, Comput. Phys. Commun. 182, 898 (2011).

${ }^{14}$ S. Pronk, S. Páll, R. Schulz, P. Larsson, P. Bjelkmar, R. Apostolov, M. R. Shirts, J. C. Smith, P. M. Kasson, D. van der Spoel, et al., Bioinformatics 29, 845 (2013).

${ }^{15}$ J. C. Phillips, R. Braun, W. Wang, J. Gumbart, E. Tajkhorshid, E. Villa, C. Chipot, R. D. Skeel, L. Kale, and K. Schulten, J. Comput. Chem. 26, 1781 (2005).

${ }^{16}$ P. Eastman, M. S. Friedrichs, J. D. Chodera, R. J. Radmer, C. M. Bruns, J. P. Ku, K. A. Beauchamp, T. J. Lane, L.-P. Wang, D. Shukla, T. Tye, M. Houston, T. Stich, C. Klein, M. R. Shirts, and V. S. Pande, J. Chem. Theory Comput. 9, 461 (2013).

${ }^{17}$ S. Páll and B. Hess, Comput. Phys. Commun. 184, 2641 (2013)

${ }^{18}$ J. E. Stone, J. C. Phillips, P. L. Freddolino, D. J. Hardy, L. G. Trabuco, and K. Schulten, J. Comput. Chem. 28, 2618 (2007).

${ }^{19}$ W. Liu, B. Schmidt, G. Voss, and W. Müller-Wittig, Comput. Phys. Commun. 179, 634 (2008).

${ }^{20}$ A. Zhmurov, R. Dima, Y. Kholodov, and V. Barsegov, Proteins 78, 2984 (2010).

${ }^{21}$ D. M. Hinckley, G. S. Freeman, J. K. Whitmer, and J. J. de Pablo, J. Chem. Phys. 139, 144903 (2013)

${ }^{22}$ J. P. K. Doye, T. E. Ouldridge, A. A. Louis, F. Romano, P. Sulc, C. Matek, B. E. K. Snodin, L. Rovigatti, J. S. Schreck, R. M. Harrison, and W. P. J. Smith, Phys. Chem. Chem. Phys. 15, 20395 (2013)

${ }^{23}$ D. C. Rapaport, Phys. Rev. Lett. 101, 186101 (2008).

${ }^{24}$ I. Coluzza, P. D. J. van Oostrum, B. Capone, E. Reimhult, and C. Dellago, Phys. Rev. Lett. 110, 075501 (2013)

${ }^{25}$ F. Romano and F. Sciortino, Nat. Commun. 3, 975 (2012).

${ }^{26}$ L. Rovigatti, F. Smallenburg, F. Romano, and F. Sciortino, ACS Nano 8, 3567 (2014)

${ }^{2}$ J. Russo, P. Tartaglia, and F. Sciortino, J. Chem. Phys. 131, 014504 (2009)

${ }^{2 \varnothing} \mathrm{L}$. Rovigatti, Role of the anisotropy in the interactions between nano- and micro-sized particles, Ph.D. thesis, Sapienza Università di Roma (2012).

${ }^{29}$ P. H. Colberg and F. Höfling, Comput. Phys. Commun. 182, 1120 (2011)

30 "See Supplementary Materials No. XXXX for the comparison of the vertex-based algorithm performance with and without Newton's third law implemented and for the benchmark comparison of oxDNA with HOOMD and LAMMPS packages for simulations of LJ system.".

${ }^{31}$ T. E. Ouldridge, A. A. Louis, and J. P. K. Doye, J. Chem. Phys. 134, 085101 (2011)

${ }^{32}$ P. Sulc, F. Romano, T. E. Ouldridge, L. Rovigatti, J. P. K. Doye, and A. A. Louis, J. Chem. Phys. 137, 135101 (2012)

${ }^{33}$ http://dna.physics.ox.ac.uk.

${ }^{34}$ W. Kob and H. C. Andersen, Phys. Rev. Lett. 73, 1376 (1994)

${ }^{35}$ E. Bianchi, R. Blaak, and C. Likos, Phys. Chem. Chem. Phys. (2011).

${ }^{36}$ S. C. Glotzer and M. J. Solomon, Nat. Mater. 6, 557 (2007).

${ }^{37}$ L. Rovigatti, W. Kob, and F. Sciortino, J. Chem. Phys. 135, $104502(2011)$

${ }^{8}$ Q. Chen, S. C. Bae, and S. Granick, Nature (2011).

${ }^{39}$ J. Russo, J. Tavares, P. Teixeira, M. T. da Gama, and F. Sciortino, J. Chem. Phys. 135, 034501 (2011).

${ }^{40}$ A. Reinhardt, A. J. Williamson, J. P. K. Doye, J. Carrete, L. M. Varela, and A. A. Louis, J. Chem. Phys. 134, 104905 (2011).

${ }^{41}$ L. Rovigatti and F. Sciortino, Mol. Phys. 109, 2889 (2011)

${ }^{42}$ T. E. Ouldridge, Coarse-Grained Modelling of DNA and DNA self-assembly, Ph.D. thesis, University of Oxford (2011).

${ }^{43}$ T. E. Ouldridge, A. A. Louis, and J. P. K. Doye, Phys. Rev. Lett. 104, 178101 (2010) 
${ }^{44}$ T. E. Ouldridge, R. L. Hoare, A. A. Louis, J. P. K. Doye, J. Bath, and A. J. Turberfield, ACS Nano 7, 2479 (2013)

${ }^{45}$ P. Šulc, T. E. Ouldridge, F. Romano, J. P. Doye, and A. A. Louis, Natural Computing (2013), 10.1007/s11047-013-9391-8

${ }^{46}$ L. Rovigatti, F. Bomboi, and F. Sciortino, J. Chem. Phys. 140, $154903(2014)$.

4'NVIDIA, "Kepler GK110 whitepaper," http://www.nvidia.com/content/PDF/kepler/NVIDIA-KeplerGK110-Architecture-Whitepaper.pdf (2012).

\section{SUPPLEMENTARY INFORMATION}

In this Supplementary information we report a comparison between oxDNA and HOOMD-blue-blue and LAMMPS, as well as absolute timings for the test cases employed in the text.

\section{COMPARISON TO HOOMD-BLUE AND LAMMPS}

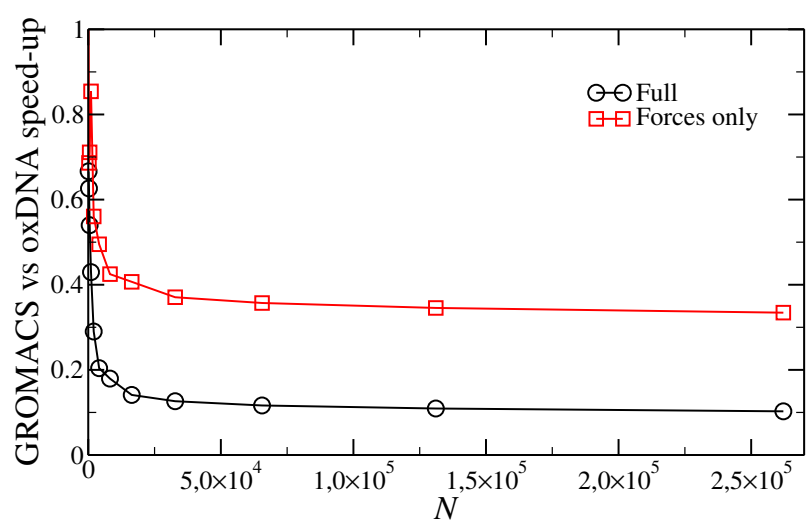

FIG. 5. Performance comparison between oxDNA and GROMACS 5.0.1. Timings are carried out on a C2070 NVIDIA GPU. We show the ratio between the average time required by oxDNA to perform an MD step over the time taken by GROMACS (in black). We also show the ratio between the average time required by the kernel computing the forces of oxDNA and of GROMACS (in red).
Since our aim is to compare parallelization algorithms, it is important to verify that the performances of our code are comparable to state-of-the-art codes that are publicly available. Figure 2 in the main text shows the comparison between oxDNA and the GPU-enabled versions of HOOMD-blue (0.11-3 version) and LAMMPS (1Feb14 version) for a standard NVE Lennard-Jones simulation at $\rho=0.1$ (vs HOOMD-blue) and $\rho=0.8442$ (vs LAMMPS) for several system sizes. Overall, the large- $N$ performances are roughly equivalent, with the largest difference being less than $20 \%$. In addition, the asymptotic performances of the three codes are very similar.

Figure 5 shows a comparison (carried out at $\rho=0.1$ ) between oxDNA and GROMACS 5.0.1. oxDNA is faster for all the considered number of particles. We stress that GROMACS uses the GPU only to calculate non-bonded forces and thus we also show the relative performance between the force-computing kernels in oxDNA and GROMACS. We note that GROMACS is not tailored to simulate short-ranged-only potentials and hence it is perhaps unsurprising that it does not perform very well in this particular test.

\section{ABSOLUTE TIMINGS AND COMPARISON OF THE VERTEX-BASED APPROACH WITH AND WITHOUT NEWTON'S THIRD LAW}

For reference, we report in Fig. 6 the absolute time that is required for each of the algorithms to perform a single molecular dynamics step with the different architectures and models. The figures include timings for edgebased approach ("edge"), vertex-based approach ("vertex") and vertex-based approach with Newton's third law implemented ("newton") for C2070, K10 and K20 architectures 

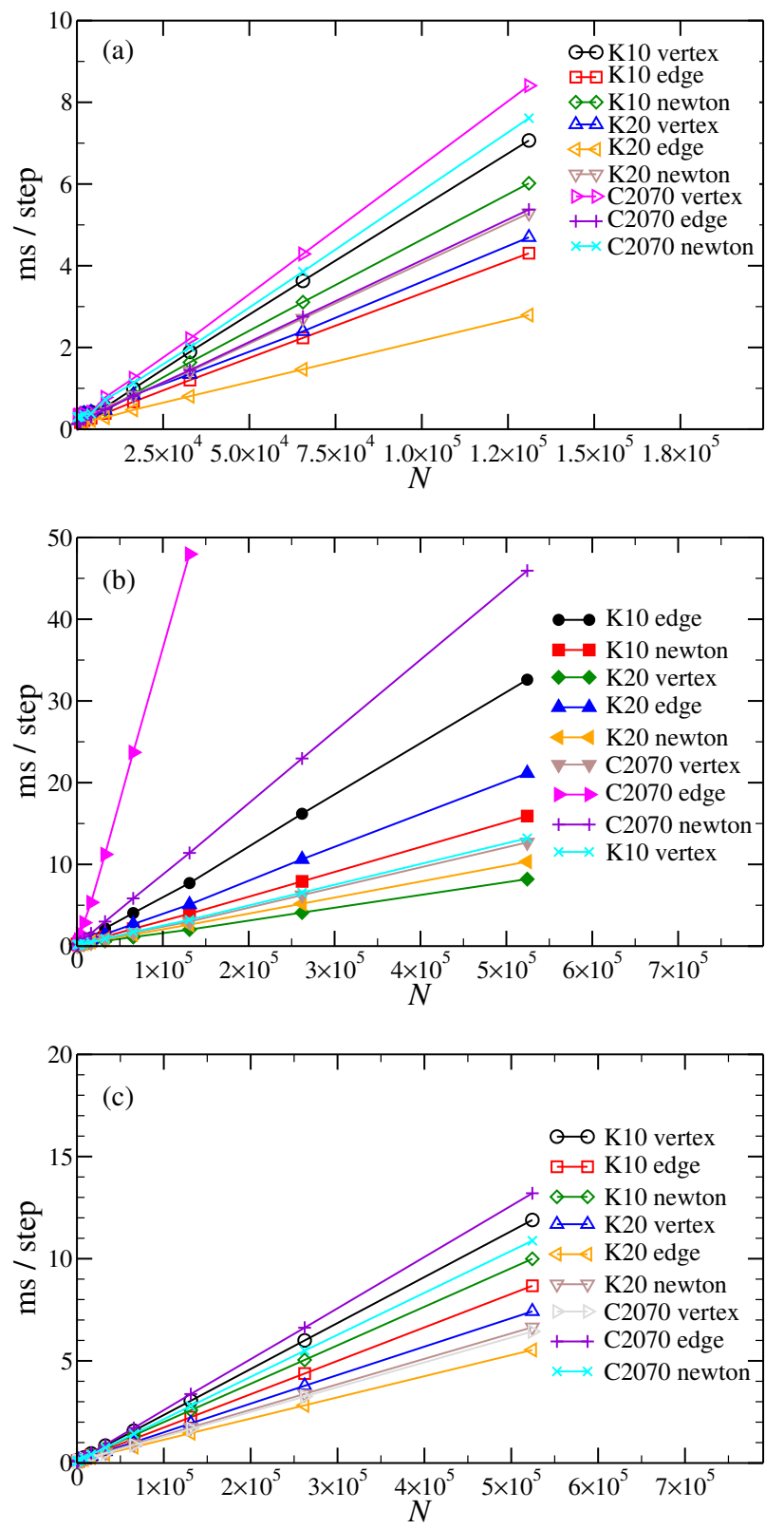

FIG. 6. Absolute timings as a function of system sizes. (a) DNA model at a double strand concentration of $2.7 \mathrm{mM}, T=$ $300 \mathrm{~K}$. (b) LJ model, $k_{B} T / \epsilon=1.8, \rho \sigma^{3}=0.5$. (c) patchy model, $k_{B} T / \epsilon=0.15, k_{B} T / \epsilon=1.8, \rho \sigma^{3}=0.5$ 